\title{
NUMERICAL ALGORITHM FOR FINDING BALANCED METRICS ON VECTOR BUNDLES*
}

\author{
REZA SEYYEDALI ${ }^{\dagger}$
}

\begin{abstract}
In [D5], Donaldson defines a dynamical system on the space of Fubini-Study metrics on a polarized compact Kähler manifold. Sano proved that if there exists a balanced metric for the polarization, then this dynamical system always converges to the balanced metric ([S]). In [DKLR], Douglas, et. al., conjecture that the same holds in the case of vector bundles. In this paper, we give an affirmative answer to their conjecture.
\end{abstract}

Key words. Holomorphic vector bundles, Gieseker stability, Balanced metrics.

AMS subject classifications. Primary 53C07; Secondary $32 \mathrm{Q} 26$

1. Introduction. In [NS], Narasimhan and Seshadri prove that a holomorphic vector bundle over a compact Riemann surface is stable if and only if it arises from an irreducible projective unitary representation of the fundamental group of the Riemann surface. Later, Donaldson proves that a holomorphic vector bundle over a smooth compact algebraic surface is poly-stable if and only if it admits a Hermitian-Einstein metric ([D1]). Uhlenbeck and Yau generalize Donaldson's result to compact Kähler manifolds of arbitrary dimension ([D2], [UY]).

Closely related to stability is the notion of balanced embeddings and balanced metrics. The idea of balanced embeddings appeared in the work of Bourguignon, Li and Yau ([BLY]). They use balanced embeddings to prove an upper bound for the first eigenvalue of the laplacian on a Kähler manifold. Also in [Zh], Zhang proves that a smooth projective variety admits a balanced embedding into some projective space if and only if it is Chow semi-stable. Later, Donaldson defines the concept of balanced metric for holomorphic vector bundles ([D3]). By a theorem of Wang, we know that a holomorphic vector bundle $E$ admits a unique (up to a positive constant) balanced metric if and only if the Gieseker point of $E$ is stable (([W, Theorem 1.1]), [PS]). On the other hand, there exists at most one balanced metric (up to a constant) provided the bundle is simple (cf. Lemma 2.7 below).

In [D5], Donaldson defines a dynamical system on the space of Fubini-Study metrics on a polarized compact Kähler manifold. Sano proved that if there exists a balanced metric for the polarization, then this dynamical system always converges to the balanced metric ([S]). In [DKLR], Douglas, et. al., conjecture that the same holds in the case of vector bundles. In this paper, we give an affirmative answer to their conjecture.

Let $(X, \omega)$ be a Kähler manifold of dimension $m$ and $E$ be a very ample holomorphic vector bundle on $X$. Let $h$ be a Hermitian metric on $E$. We can define a $L^{2}$-inner product on $H^{0}(X, E)$ by

$$
\langle s, t\rangle=\int_{X} h(s, t) \frac{\omega^{m}}{m !} .
$$

\footnotetext{
*Received April 12, 2008; accepted for publication April 7, 2009.

${ }^{\dagger}$ Department of Mathematics, University of California, Irvine - Irvine, CA 92697-3875, USA (rseyyeda@math.uci.edu).
} 
Let $s_{1}, \ldots, s_{N}$ be an orthonormal basis for $H^{0}(X, E)$ with respect to this $L^{2}$-inner product. The Bergman kernel of $h$ is defined by

$$
B(h)=\sum s_{i} \otimes s_{i}^{*_{h}} .
$$

Note that $B(h)$ does not depend on the choice of the orthonormal basis $s_{1}, \ldots, s_{N}$. A metric $h$ is called balanced if $B(h)$ is a constant multiple of the identity.

Let $K$ and $M$ be the space of Hermitian metrics on $E$ and Hermitian inner product on $H^{0}(X, E)$ respectively. Following Donaldson ([D4]), one can define the following maps

$$
\begin{aligned}
\text { Hilb }: K & \rightarrow M, \quad h \mapsto \operatorname{Hilb}(h) \\
\langle s, t\rangle_{\operatorname{Hilb}(h)} & =\frac{N}{V r} \int\langle s(x), t(x)\rangle_{h} \frac{\omega^{m}}{m !},
\end{aligned}
$$

where $N=\operatorname{dim}\left(H^{0}(X, E)\right)$ and $V=\operatorname{Vol}(X, \omega)$. Note that Hilb only depends on the volume form $\omega^{m} / m$ !.

- For the metric $H \in M, F S(H)$ is the unique metric on $E$ such that $\sum s_{i} \otimes s_{i}^{*} F(H)=I$, where $s_{1}, \ldots, s_{N}$ is an orthonormal basis for $H^{0}(X, E)$ with respect to $H$. This gives the map $F S: M \rightarrow K$.

- Define

$$
T: M \rightarrow M
$$

$T(H)=$ Hilb $\circ F S(H)$. This map $T$ is called the generalized $T$-operator in [DKLR].

It is easy to see that a metric $h$ is balanced if and only if $\operatorname{Hilb}(h)$ is a fixed point of the map $T$. The main theorem of this paper is the following

Theorem 1.1. Suppose that $E$ is simple and admits a balanced metric. Then for any hermitian inner product $H_{0}$ on $H^{0}(X, E)$, the sequence $T^{n}\left(H_{0}\right)$ converges to $H_{\infty}$ as $n \rightarrow \infty$, where $H_{\infty}$ is a balanced metric on $E$.

Our proof follows Sano's argument in $[\mathrm{S}]$ with the necessary modifications for the bundle case.

In order to prove the theorem, we consider the functional $Z$ that is used by Wang ([W]) and Phong, Sturm ([PS]) in order to study the existence and uniqueness of balanced metrics on holomorphic vector bundles. The key property of this functional is that its critical points are balanced metrics. In the second section we recall some properties of the functionals $Z$ and $\widetilde{Z}$. In the second section, we give an appropriate notion of boundedness for subsets of $M$, defined in [S]. With this definition, any bounded sequence has a convergent subsequence after a suitable rescaling of the sequence. Therefore in order to prove that the sequence $H_{n}=T^{n}(H)$ converges, we need to show that $H_{n}$ is bounded. On the other hand, existence of a balanced metric implies that $\widetilde{Z}$ is bounded from below and proper in a suitable sense. Hence it shows that $\widetilde{Z}\left(H_{n}\right)$ is bounded. Now properness of $\widetilde{Z}$ implies that $H_{n}$ is bounded.

Acknowledgements. I am sincerely grateful to Richard Wentworth for introducing me the subject and many helpful discussions and suggestions on the subject. I would also like to thank him for all his help, support and encouragement. 
2. Balanced metrics on vector bundles. As before, let $(X, \omega)$ be a Kähler manifold and $E$ be a very ample holomorphic vector bundle on $X$. Using global sections of $E$, we can map $X$ into $G\left(r, H^{0}(X, E)^{*}\right)$. Indeed, for any $x \in X$, we have the evaluation map $H^{0}(X, E) \rightarrow E_{x}$, which sends $s$ to $s(x)$. Since $E$ is globally generated, this map is a surjection. So its dual is an inclusion of $E_{x}^{*} \hookrightarrow H^{0}(X, E)^{*}$, which determines a $r$-dimensional subspace of $H^{0}(X, E)^{*}$. Therefore we get an embedding $i: X \hookrightarrow G\left(r, H^{0}(X, E)^{*}\right)$. Clearly we have $i^{*} U_{r}=E^{*}$, where $U_{r}$ is the tautological vector bundle on $G\left(r, H^{0}(X, E)^{*}\right)$, i.e. at any $r$-plane in $G\left(r, H^{0}(X, E)^{*}\right)$, the fibre of $U_{r}$ is exactly that $r$-plane. A choice of basis for $H^{0}(X, E)$ gives an isomorphism between $G\left(r, H^{0}(X, E)^{*}\right)$ and the standard $G(r, N)$, where $N=\operatorname{dim} H^{0}(X, E)$. We have the standard Fubini-Study hermitian metric on $U_{r}$, so we can pull it back to $E$ and get a hermitian metric on $E$. Using $i^{*} h_{F S}$ and $\omega$, we get an $L^{2}$ inner product on $H^{0}(X, E)$. The embedding is called balanced if $\int_{X}\left\langle s_{i}, s_{j}\right\rangle \frac{\omega^{m}}{m !}=C \delta_{i j}$. We can formulate this definition in terms of maps Hilb and $F S$.

Definition 2.1. A balanced metric on $E$ is a pair $\left(h^{*}, H^{*}\right)$ so that

$$
\operatorname{Hilb}\left(h^{*}\right)=H^{*}, F S\left(H^{*}\right)=h^{*}
$$

Fixing a nonzero element $\Theta \in \bigwedge^{N} H^{0}(X, E)$, We can define the determinant of any element in $M$. Thus we can define a map

$$
\log \operatorname{det}: M \rightarrow \mathbb{R}
$$

A different choice of $\Theta$ only changes this map by an additive constant. Also, we define a functional $I: K \rightarrow \mathbb{R}$ again unique up to an additive constant. Fix a background metric $h_{0}$ and consider a path $h_{t}=e^{\phi_{t}} h_{0}$ in $K$ then

$$
\frac{d I}{d t}=\int_{X} \operatorname{tr}(\dot{\phi}) \mathrm{dVol}_{\omega}
$$

This functional is a part of Donaldson's functional. We define:

$$
Z=-I \circ F S: M \rightarrow \mathbb{R}
$$

We have the following scaling identities:

$$
\begin{gathered}
\operatorname{Hilb}\left(e^{\alpha} h\right)=e^{\alpha} \operatorname{Hilb}(h), \\
F S\left(e^{\alpha} h\right)=e^{\alpha} F S(h), \\
I\left(e^{\alpha} h\right)=I(h)+\alpha r V,
\end{gathered}
$$

where $\alpha$ is a real number.

Following Donaldson, define:

$$
\widetilde{Z}=Z+\frac{r V}{N} \log \operatorname{det} .
$$

So $\widetilde{Z}$ is invariant under constant scaling of the metric. 
This functional $Z$ is studied by Wang in [W] and Phong and Sturm in [PS]. They consider this as a functional on $S L(N) / S U(N)$. In order to see this, we observe that there is a correspondence between $M$ and $G L(N) / U(N)$. Fix an element $H_{0} \in M$ and an orthonormal basis $s_{1}, \ldots, s_{N}$ for $H^{0}(X, E)$ with respect to $H_{0}$. Now for any $H \in M$ we assign $\left[H\left(s_{i}, s_{j}\right)\right] \in G L(N)$. Notice that a change of the orthonormal basis only changes this matrix by multiplication by elements of $U(N)$. So we get a well define element of $G L(N) / U(N)$. The subset

$$
M_{0}=\left\{H \in M \mid \operatorname{det}\left[H\left(s_{i}, s_{j}\right)\right]=1\right\}
$$

corresponds to $S L(N) / S U(N)$.

Recall the definition of the Gieseker point of the bundle $E$. We have a natural map

$$
T(E): \bigwedge^{r} H^{0}(X, E) \rightarrow H^{0}(X, \operatorname{det}(E))
$$

which for any $s_{1}, \ldots, s_{r}$ in $H^{0}(X, E)$ is defined by

$$
T(E)\left(s_{1} \wedge \ldots \wedge s_{r}\right)(x)=s_{1}(x) \wedge \ldots \wedge s_{r}(x) .
$$

Since $E$ is globally generated, $T(E)$ is surjective. We can view $T(E)$ as an element of $\operatorname{Hom}\left(\bigwedge^{r} H^{0}(X, E), H^{0}(X, \operatorname{det}(E))\right)$. This is called the Gieseker point of $E([\mathrm{G}])$. Notice that fixing a basis for $H^{0}(X, E)$ gives an isomorphism between $\bigwedge^{r} H^{0}(X, E)$ and $\bigwedge^{r} \mathbb{C}^{N}$. Hence, there is a natural action of $G L(N)$ on $\operatorname{Hom}\left(\bigwedge^{r} H^{0}(X, E), H^{0}(X, \operatorname{det}(E))\right)$. Phong-Sturm ([PS]) and Wang ([W]) prove that $Z$ is convex along geodesics of $S L(N) / S U(N)$ and its critical points are corresponding to balanced metrics on $E$. Phong and Sturm prove the following

THEOREM 2.1 ([PS, Theorem 2]). There exists a $S U(N)$ - invariant norm $\|$.$\| on$ $\operatorname{Hom}\left(\bigwedge^{r} H^{0}(X, E), H^{0}(X, \operatorname{det}(E))\right)$ such that for any $\sigma \in S L(N)$

$$
Z(\sigma)=\log \frac{\|\sigma \cdot T(E)\|^{2}}{\|T(E)\|^{2}}
$$

REMARK 2.2. In [W], Wang proves a slightly weaker version of Theorem 2.1. He proves that for any norm $\|$.$\| on \operatorname{Hom}\left(\bigwedge^{r} H^{0}(X, E), H^{0}(X, \operatorname{det}(E))\right)$, there exists positive constants $c$ and $c^{\prime}$ such that

$$
Z(\sigma) \geq c \log \|\sigma \cdot T(E)\|^{2}+c^{\prime}
$$

Theorem 2.3 ([W, Lemma 3.5], [PS, Lemma 2.2]). The functional $Z$ is convex along geodesics of $M$.

The Kempf-Ness theorem $([\mathrm{KN}])$ shows that $Z$ is proper and bounded from below if $T(E)$ is stable under the action of $S L(N)$.

The following is an immediate consequence of the above theorem and the fact that balanced metrics are critical points of $Z$. Also notice that $\widetilde{Z}$ is invariant under the scaling of a metric by a positive real number. 
TheOREM 2.4. Assume that $H_{0}$ is a balanced metric on $E$. Then $\left.\widetilde{Z}\right|_{M_{0}}$ is proper and bounded from below. Moreover $\widetilde{Z}(H) \geq \widetilde{Z}\left(H_{0}\right)$ for any $H \in M$.

Lemma 2.5. For any $H \in M$, we have

$$
\operatorname{Tr}\left(T(H) H^{-1}\right)=N
$$

Proof. Let $h=F S(H)$ and let $s_{1}, \ldots, s_{N}$ be an $H$-orthonormal basis. We have,

$$
\sum s_{i} \otimes s_{i}^{*_{h}}=I
$$

Therefore,

$$
r=\operatorname{Tr}\left(\sum s_{i} \otimes s_{i}^{* h}\right)=\sum\left|s_{i}\right|_{h}^{2} .
$$

Integrating the above equation implies the result.

Lemma 2.6. For any $H \in M$,

- $Z(H) \geq Z(T(H))$.

- $\log \operatorname{det}(H) \geq \log \operatorname{det}(T(H))$.

- $\widetilde{Z}(H) \geq \widetilde{Z}(T(H))$.

Proof. Put $h=F S(H), H^{\prime}=$ Hilb $\circ F S(H)$ and $h^{\prime}=F S\left(H^{\prime}\right)=e^{\varphi} h$. Let $s_{1}, \ldots, s_{N}$ be an $H^{\prime}$-orthonormal basis. We have,

$$
\sum s_{i} \otimes s_{i}^{* h}=e^{-\varphi} .
$$

Hence,

$$
\begin{aligned}
\int_{X} \operatorname{tr}(-\varphi) & =\int_{X} \log \operatorname{det}\left(e^{-\varphi}\right) \leq \int_{X} \log \left(\frac{\operatorname{tr}\left(e^{-\varphi}\right)}{r}\right)^{r} \\
& =r \int_{X} \log \left(t r\left(e^{-\varphi}\right)\right)-r V \log r \\
& \leq r V \log \left(\frac{1}{V} \int_{X} \operatorname{tr}\left(e^{-\varphi}\right)\right)-r V \log r \\
& =r V \log \left(\frac{1}{V} \int_{X} \sum\left|s_{i}\right|_{h}^{2}\right)-r V \log r=0
\end{aligned}
$$

This shows the first inequality. For the second one, Lemma 2.5 implies that $\operatorname{tr}\left(H^{\prime} H^{-1}\right)=N$. Using the arithmetic -geometric mean inequality, we get

$$
\operatorname{det}\left(H^{\prime} H^{-1}\right)^{\frac{1}{N}} \leq \frac{\operatorname{tr}\left(H^{\prime} H^{-1}\right)}{N}=1 .
$$

This implies that $\log \operatorname{det}\left(H^{\prime} H^{-1}\right) \leq 0$. The third inequality is obtained by summing up the first two. $\mathrm{C}$

A bundle $E$ is called simple if $A u t(E) \simeq \mathbb{C}^{*}$. We will need the following

Lemma 2.7. Suppose that $E$ is simple and admits a balanced metric. Then the balanced metric is unique up to a positive constant.

Proof. Since $\operatorname{det}(H)^{-1 / N} H \in M_{0}$ for any $H \in M$, it suffices to prove that a balanced metric in $M_{0}$ is unique. Let $H_{\infty} \in M_{0}$ be a balanced metric on $E$ and 
$s_{1}, \ldots, s_{N}$ be an orthonormal basis of $H^{0}(X, E)$ with respect to $H_{\infty}$. This basis gives an embedding $\iota: X \rightarrow G r(r, N)$ such that $\iota^{*} U_{r}=E$, where $U_{r} \rightarrow G r(r, N)$ is the universal bundle over the Grassmannian. Assume that $H$ is another element of $M_{0}$. Therefore, there exists an element $a \in s u(N)$ such that $e^{i a} \cdot H_{\infty}=H$. Then $\left\{e^{i t a}\right\}$ gives a one parameter family of automorphism of $\left(G r(r, N), U_{r}\right)$ and therefore a one parameter family in $\operatorname{Aut}(X, E)$. From lemma 3.5 in $[\mathrm{W}]$, we have

$$
\frac{d^{2}}{d t^{2}} Z\left(e^{i t a}\right)=\int_{\iota(X)}\|\tilde{a}\|^{2} d v o l_{X}
$$

where $\tilde{a}$ is the vector field on $\operatorname{Gr}(r, N)$ generated by the infinitesimal action of $a$ and $\|\tilde{a}\|$ is the Fubini-Study norm of $\widetilde{a}$. Suppose that $H$ is a balanced metric. Therefore it is a minimum for the functional $Z$. This implies that

$$
\frac{d^{2}}{d t^{2}} Z\left(e^{i t a}\right)=0
$$

and hence by (2.4) that $\left.\tilde{a}\right|_{\iota(X)} \equiv 0$. This implies that the one parameter family $\left\{e^{i t a}\right\}$ fixes $\iota(X)$ pointwise and therefore the one parameter family $\left\{e^{i t a}\right\}$ is a one parameter family of endomorphisms of $E$. By the simplicity of $E, e^{i a}$ as an endomorphism of $E$ must be a constant scalar of the identity. Therefore $H$ is a constant multiple of $H_{\infty}$. It implies that $H=H_{\infty}$ since $H \in M_{0}$.

3. Proof of Theorem 1.1. In this section, we closely follow Sano's argument in ([S, Section 3]). Let $s_{1}, \ldots, s_{N}$ be a basis for $H^{0}(E)$. With this basis, we can view elements of $M$ as $N \times N$ matrices. Now using this identification, we state the following definition introduced in Sano ([S]).

Definition 3.1. A subset $U \subseteq M$ is called bounded if there exists a number $R>1$, satisfying the following: For any $H \in U$, there exists a positive number $\gamma_{H}$ so that

$$
\frac{\gamma_{H}}{R} \leq \min \frac{|H(\xi)|}{|\xi|} \leq \max \frac{|H(\xi)|}{|\xi|} \leq \gamma_{H} R
$$

Note that boundedness does not depend on the choice of the basis. Also notice that $\min |H(\xi)| /|\xi|$ is the smallest eigenvalue of the matrix $\left[H\left(s_{i}, s_{j}\right)\right]$ and $\max |H(\xi)| /|\xi|$ is the largest eigenvalue of the matrix $\left[H\left(s_{i}, s_{j}\right)\right]$.

From the definition, one can see that $U$ is bounded if and only if there exists $R>1$ satisfying the following: For any $H \in U$, there exists a positive number $\gamma_{H}$ so that

$$
\begin{gathered}
\left\|\left[H\left(s_{i}, s_{j}\right)\right]\right\|_{o p} \leq \gamma_{H} R, \\
\left\|\left[H\left(s_{i}, s_{j}\right)\right]^{-1}\right\|_{o p} \leq \gamma_{H}^{-1} R .
\end{gathered}
$$

Proposition 3.1. Any bounded sequence $H_{i}$ has a subsequence $H_{n_{i}}$ such that $\gamma_{n_{i}}^{-1} H_{n_{i}}$ converges to some point in $M$. Here $\gamma_{i}=\gamma_{H_{i}}$ in Definition 3.1.

Proof. The sequence $\gamma_{n_{i}}^{-1} H_{n_{i}}$ is a bounded sequence in the space of $N \times N$ matrices with respect to the standard topology. Hence the proposition follows from the fact that the closure of bounded sets are compact. 
Notice that the standard topology on the space of $N \times N$ matrices is induced by the standard Euclidean norm. Since all norms on a finite dimensional vector space are equivalent, we can use the operator norm. Therefore a sequence $\left\{H_{\alpha}\right\}$ in $M$ converges to $H \in M$ if and only if

$$
\left|\left[H_{\alpha}\left(s_{i}, s_{j}\right)\right]-\left[H\left(s_{i}, s_{j}\right)\right]\right|_{o p} \rightarrow 0 \quad \text { as } \quad \alpha \rightarrow 0 .
$$

Lemma 3.2. The set $U \subseteq M$ is bounded if and only if there exists a number $\widetilde{R}>1$ so that for any $H \in U$, we have

$$
\frac{1}{\widetilde{R}} \leq \min \frac{|\widetilde{H}(\xi)|}{|\xi|} \leq \max \frac{|\widetilde{H}(\xi)|}{|\xi|} \leq \widetilde{R},
$$

where $\widetilde{H}=(\operatorname{det}(H))^{-\frac{1}{N}} H$.

Proof. Assume that $U$ is bounded. By definition there exists a number $R>1$, satisfying (3.1). Let $H$ be an element of $U$. Without loss of generality we can assume that $H\left(s_{i}, s_{j}\right)=e^{\lambda_{i}} \delta_{i j}$ and $\lambda_{1} \leq \ldots \leq \lambda_{N}$. For any $i$, we have

$$
\frac{\gamma_{H}}{R} \leq e^{\lambda_{i}} \leq \gamma_{H} R
$$

This implies that $\gamma_{H} \leq R e^{\lambda_{i}}$ and $\gamma_{H} \geq R^{-1} e^{\lambda_{i}}$. Therefore

$$
e^{\lambda_{N}} \leq \gamma_{H} R \leq R^{2} e^{\lambda_{i}}
$$

and

$$
e^{\lambda_{1}} \geq \gamma_{H} R^{-1} \geq R^{-2} e^{\lambda_{i}}
$$

for any $1 \leq i \leq N$. Hence

$$
(\operatorname{det}(H))^{\frac{-1}{N}} e^{\lambda_{N}}=e^{\lambda_{N}-\frac{\sum \lambda_{i}}{N}}=\left(\prod e^{\lambda_{N}-\lambda_{i}}\right)^{\frac{1}{N}} \leq R^{2} .
$$

and

$$
(\operatorname{det}(H))^{\frac{-1}{N}} e^{\lambda_{1}}=e^{\lambda_{1}-\frac{\sum \lambda_{i}}{N}}=\left(\prod e^{\lambda_{1}-\lambda_{i}}\right)^{\frac{1}{N}} \geq R^{-2} .
$$

Put $\widetilde{R}=R^{2}$.

The converse is followed by letting $\gamma_{H}=\operatorname{det}(H)^{-1 / N}$.

Let $H_{0}$ be an element in $M$. Define the sequence $\left\{H_{n}\right\}$ by $H_{n}=T\left(H_{n-1}\right)$.

Lemma 3.3. If $\left\{H_{n}\right\}$ is a bounded sequence in $M$, then $\operatorname{det}\left(H_{n}\right)$ is bounded and

$$
\operatorname{det}\left(H_{n+1} H_{n}^{-1}\right) \rightarrow 1 \text { as } n \rightarrow \infty .
$$

In particular $\operatorname{det}\left(H_{n}\right)$ converges to a positive number.

Proof. $\widetilde{Z}\left(H_{n}\right)$ is bounded since the sequence $\left\{H_{n}\right\}$ is bounded. On the other hand, lemma 2.6 implies that the sequences $Z\left(H_{n}\right)$ and $\log \operatorname{det}\left(H_{n}\right)$ are decreasing. So, $\log \operatorname{det}\left(H_{n}\right)$ is bounded and decreasing. Hence, it converges to some real number. This implies that $\operatorname{det}\left(H_{n+1} H_{n}^{-1}\right) \rightarrow 1$ as $n \rightarrow \infty$. $\square$ 
Lemma 3.4. Assume $\left\{H_{n}\right\}$ is a bounded sequence in $M$. Let $H$ be a fixed element of $M$ and $s_{1}^{(l)}, . . s_{N}^{(l)}$ be an orthonormal basis with respect to $H_{l}$ so that the matrix $\left[H\left(s_{i}^{(l)}, s_{j}^{(l)}\right)\right]$ is diagonal. Then

$$
\frac{N}{V r} \int_{X}\left|s_{i}^{l}\right|_{h_{l}}^{2} d v o l_{X} \rightarrow 1 \quad \text { as } \quad l \rightarrow \infty
$$

where $h_{n}=F S\left(H_{n}\right)$.

Proof. Let $\hat{s}_{1}^{(l)}, . . \hat{s}_{N}^{(l)}$ be an orthonormal basis with respect to $H_{l}$ so that $H_{l+1}\left(\hat{s}_{i}^{(l)}, \hat{s}_{j}^{(l)}\right)$ is diagonal. Hence

$$
\operatorname{det}\left[H_{l+1}\left(\hat{s}_{i}^{(l)}, \hat{s}_{j}^{(l)}\right)\right]=\prod_{i=1}^{N} H_{l+1}\left(\hat{s}_{i}^{(l)}, \hat{s}_{1}^{(l)}\right) .
$$

Lemma 3.3 implies that

$$
\operatorname{det}\left[H_{l+1}\left(\hat{s}_{i}^{(l)}, \hat{s}_{j}^{(l)}\right)\right] \rightarrow 1 .
$$

On the other hand Lemma 2.6 implies that

$$
\operatorname{tr}\left[H_{l+1}\left(\hat{s}_{i}^{(l)}, \hat{s}_{j}^{(l)}\right)\right]=N .
$$

We define $A_{l}(i)=H_{l+1}\left(\hat{s}_{i}^{(l)}, \hat{s}_{i}^{(l)}\right)$. Therefore,

$$
\begin{gathered}
\prod_{i=1}^{N} A_{l}(i) \rightarrow 1 \quad \text { as } \quad l \rightarrow \infty, \\
\sum_{i=1}^{N} A_{l}(i)=N, \quad \text { for any } 1 \leq l \leq N .
\end{gathered}
$$

We claim that for any $i$,

$$
A_{l}(i) \rightarrow 1 \quad \text { as } \quad l \rightarrow \infty .
$$

Suppose not, then there exist an $\alpha, 1 \leq \alpha \leq N$, a positive number $\epsilon>0$ and a subsequence $\left\{A_{l_{q}}(\alpha)\right\}$ such that

$$
\left|A_{l_{q}}(\alpha)-1\right| \geq \epsilon .
$$

On the other hand, (3.3) implies that $A_{l}(i) \leq N$ since $A_{l}(i) \geq 0$ and therefore the sequences $\left\{A_{l_{q}}(i)\right\}$ are bounded for any $1 \leq i \leq N$. Hence there exist nonnegative numbers $A(1), \ldots A(N)$ and a subsequence $\left\{l_{q_{j}}\right\}$ so that

$$
A_{l_{q_{j}}}(i) \rightarrow A(i) \quad \text { as } \quad j \rightarrow \infty .
$$

Therefore, (3.2), (3.3) and (3.6) imply that

$$
\prod_{i=1}^{N} A(i)=1 \quad \text { and } \quad \sum_{i=1}^{N} A(i)=N .
$$


By arithmetic-geometric mean inequality, we always have

$$
\left(\prod_{i=1}^{N} A(i)\right)^{\frac{1}{N}} \leq \frac{1}{N} \sum_{i=1}^{N} A(i)
$$

and equality holds if and only if all $A_{i}$ 's are equal. Since equality holds in this case, we conclude that $A(1)=\ldots=A(N)=1$. In particular

$$
A_{l_{q_{j}}}(\alpha) \rightarrow 1 \quad \text { as } \quad j \rightarrow \infty
$$

which contradicts (3.5). This implies that $H_{l+1}\left(\hat{s}_{i}^{(l)}, \hat{s}_{i}^{(l)}\right) \rightarrow 1$ for all $i$.

On the other hand, there exists $\left[a_{i j}^{l}\right] \in U(N)$ such that $s_{i}^{(l)}=\sum_{j=1}^{N} a_{i j} \hat{s}_{j}^{l}$. Since $U(N)$ is compact, we can find a subsequence of $\left[a_{i j}^{l}\right]$ which converges to an element of $U(N)$. Without loss of generality, we can assume that there exists $\left[a_{i j}\right] \in U(N)$ such that $a_{i j}^{l} \rightarrow a_{i j}$ as $l \rightarrow \infty$. We have,

$$
H_{l+1}\left(s_{i}^{(l)}, s_{i}^{(l)}\right)=\sum a_{i j}^{l} \overline{a_{i k}^{l}} H_{l+1}\left(\hat{s}_{j}^{(l)}, \hat{s}_{k}^{(l)}\right) \rightarrow \sum_{j=1}^{N}\left|a_{i j}\right|^{2}=1 .
$$

Proposition 3.5 (cf. [S, Proposition 3.2]). If $\left\{H_{n}\right\}$ is a bounded sequence in $M$, then for any $H \in M$ and any $\epsilon>0$,

$$
\widetilde{Z}(H) \leq \widetilde{Z}\left(H_{n}\right)-\epsilon,
$$

for sufficiently large $n$.

Proof. Let $s_{1}^{(l)}, \ldots, s_{N}^{(l)}$ be an orthonormal basis with respect to $H_{l}$ such that $H\left(s_{i}^{(l)}, s_{j}^{(l)}\right)=\delta_{i j} e^{\lambda_{i}^{(l)}}$. Fix a positive integer $l$. Define $H_{t}\left(s_{i}^{(l)}, s_{i}^{(l)}\right)=\delta_{i j} e^{t \lambda_{i}^{(l)}}$. We have $H_{0}=H_{l}$ and $H_{1}=H$. Let $f_{l}(t)=f(t)=\widetilde{Z}\left(H_{t}\right)$. We have

$$
\begin{aligned}
f(1)-f(0) & =\int_{0}^{1} f^{\prime}(t) d t=\int_{0}^{1}\left(f^{\prime}(0)+\int_{0}^{t} f^{\prime \prime}(s) d s\right) d t \\
& =f^{\prime}(0)+\int_{0}^{1} \int_{0}^{1} f^{\prime \prime}(s) d s d t \geq f^{\prime}(0),
\end{aligned}
$$

since $\widetilde{Z}$ is convex along geodesics. On the other hand, we have

$$
\begin{aligned}
f^{\prime}(t) & =\frac{d}{d t}\left(-I\left(F S\left(H_{t}\right)\right)+\frac{V r}{N} \log \operatorname{det}\left(H_{t}\right)\right) \\
& =-\int_{X} \frac{d}{d t}\left(F S\left(H_{t}\right)\right) d v o l_{X}+\frac{V r}{N} \sum \lambda_{i}^{(l)} .
\end{aligned}
$$

Therefore,

$$
f_{l}^{\prime}(0)=-\int_{X}\left(\sum \lambda_{i}^{(l)}\left|s_{i}^{(l)}\right|_{h_{l}}^{2}\right) d v o l_{X}+\frac{V r}{N} \sum \lambda_{i}^{(l)},
$$

where $h_{l}=F S\left(H_{l}\right)$. 
We have that $e^{\frac{-\lambda_{1}^{(l)}}{2}} s_{1}^{(l)}, \ldots, e^{\frac{-\lambda_{N}^{(l)}}{2}} s_{N}^{(l)}$ is an orthonormal basis with respect to $H$ for any $l$. Hence Lemma 3.2 implies that there exists $R>1$ so that

$$
\frac{\left(\operatorname{det}\left(H_{l}\right)\right)^{\frac{1}{N}}}{R}<H_{l}\left(e^{\frac{-\lambda_{i}^{(l)}}{2}} s_{i}^{(l)}, e^{\frac{-\lambda_{i}^{(l)}}{2}} s_{1}^{(i)}\right)<\left(\operatorname{det}\left(H_{l}\right)\right)^{\frac{1}{N}} R
$$

for any $i$ and $l$. Therefore

$$
\frac{1}{N} \log \left(\operatorname{det}\left(H_{l}\right)\right)-\log R<-\lambda_{i}^{(l)}<\frac{1}{N} \log \left(\operatorname{det}\left(H_{l}\right)\right)+\log R .
$$

This implies that $\left\{\lambda_{i}^{(l)}\right\}$ is bounded since $\left\{\operatorname{det}\left(H_{l}\right)\right\}$ is bounded by Lemma 3.3. Hence (3.8) implies that $f_{l}^{\prime}(0) \longrightarrow 0$, as $l \longrightarrow \infty$.

Corollary 3.6. If $\left\{H_{n}\right\}$ is a bounded sequence in $M$, then

$$
\widetilde{Z}\left(H_{n}\right) \longrightarrow \inf \{\widetilde{Z}(H) \mid H \in M\} .
$$

Proof of Theorem 1.1. As before, fix $H_{0} \in M$ and an orthonormal basis $s_{1}, \ldots, s_{N}$ for $H^{0}(X, E)$ with respect to the metric $H_{0}$. As in Section 2, let

$$
M_{0}=\left\{H \in M \mid \operatorname{det}\left[H\left(s_{i}, s_{j}\right)\right]=1\right\} .
$$

Assume that there exists a balanced metric $H_{\infty}$ on $E$. Since the balanced metric is unique up to a positive constant, there exists a unique balanced metric $H_{\infty} \in M_{0}$. As before, for any $H \in M$, we define

$$
\widetilde{H}=(\operatorname{det} H)^{-\frac{1}{N}} H
$$

Clearly $\widetilde{H} \in M_{0}$ and

$$
\widetilde{Z}(\widetilde{H})=\widetilde{Z}(H)=Z(\widetilde{H}) .
$$

Since there exists a balanced metric on $E$, theorem 2.4 implies that the functional $Z_{\mid M_{0}}$ is proper and bounded from below. Hence the sequence $Z\left(\widetilde{H}_{n}\right)$ is a bounded sequence in $\mathbb{R}$ since the sequence $\widetilde{Z}\left(H_{n}\right)=Z\left(\widetilde{H_{n}}\right)$ is decreasing. Therefore the sequence $\left\{\widetilde{H_{n}}\right\}$ is bounded in $M_{0}$ since $Z_{\mid M_{0}}$ is proper. We claim that

$$
\widetilde{H_{n}} \longrightarrow H_{\infty} \quad \text { as } n \rightarrow \infty .
$$

Suppose that the sequence $\left\{\widetilde{H_{n}}\right\}$ does not converge to $H_{\infty}$. Then there exists $\epsilon>0$ and a subsequence $\left\{H_{n_{j}}\right\}$ such that

$$
\left\|\widetilde{H_{n_{j}}}-H_{\infty}\right\|_{o p} \geq \epsilon .
$$

On the other hand, we know that the sequence $\left\{\widetilde{H_{n_{j}}}\right\}$ is bounded. Therefore there exist a subsequence $\left\{\widetilde{H_{n_{j_{q}}}}\right\}$ and an element $\widehat{H} \in M$ such that

$$
\widetilde{H_{n_{j_{q}}}} \rightarrow \widehat{H} \quad \text { as } \quad q \rightarrow \infty
$$

Therefore,

$$
1=\operatorname{det}\left[\widetilde{H_{n_{j_{q}}}}\left(s_{\alpha}, s_{\beta}\right)\right] \rightarrow \operatorname{det}\left[\widehat{H}\left(s_{\alpha}, s_{\beta}\right)\right] \quad \text { as } \quad q \rightarrow \infty
$$


which implies that $\widehat{H} \in M_{0}$. Now, corollary 3.6 implies that

$$
\widetilde{Z}\left(\widetilde{H_{n_{j_{q}}}}\right)=\widetilde{Z}\left(H_{n_{j_{q}}}\right) \longrightarrow \inf \{\widetilde{Z}(H) \mid H \in M\} .
$$

Hence,

$$
\widetilde{Z}(\widehat{H})=\inf \{\widetilde{Z}(H) \mid H \in M\} .
$$

This implies that $\widehat{H}$ is a balanced metric and therefore $H_{\infty}=\widehat{H}$ by lemma 2.7. This contradicts (3.9). Thus $\widetilde{H_{n}} \longrightarrow H_{\infty}$ as $q \rightarrow \infty$.

Now lemma 3.3 implies that $\log \operatorname{det}\left(H_{n}\right)$ is bounded. The sequence $\left\{\log \operatorname{det}\left(H_{n}\right)\right\}$ is bounded and decreasing. Therefore there exists $b \in \mathbb{R}$ such that

$$
\log \operatorname{det}\left(H_{n}\right) \rightarrow b \quad \text { as } \quad n \rightarrow \infty .
$$

Hence $\operatorname{det}\left(H_{n}\right)$ converges to the positive real number $e^{b}$. Thus

$$
H_{n} \longrightarrow e^{\frac{-b}{N}} H_{\infty} \quad \text { as } \quad n \rightarrow \infty .
$$

\section{REFERENCES}

[BLY] J.-P. Bourguignon, P. Li And S.-T. YAu, Upper bound for the first eigenvalue of algebraic submanifolds, Comment. Math. Helv., 69:2 (1994), pp. 199-207.

[D1] S. K. Donaldson, Anti self-dual Yang-Mills connections over complex algebraic surfaces and stable vector bundles, Proc. London Math. Soc. (3), 50:1 (1985), pp. 1-26.

[D2] S. K. Donaldson, Infinite determinants, stable bundles and curvature, Duke Math. J., 54:1 (1987), pp. 231-247.

[D3] S. Donaldson, Geometry in Oxford c. 1980-85, Asian J. Math., 3:1 (1999), pp. xliii-xlvii.

[D4] S. K. Donaldson, Scalar curvature and projective embeddings. II, Q. J. Math. 56:3 (2005), pp. $345-356$.

[D5] S. K. Donaldson, Some numerical results in complex differential geometry, arXiv:math/0512625v1 [math.DG].

[G] D. GIESEKER, On the moduli of vector bundles on an algebraic surface, Ann. of Math. (2), 106:1 (1977), pp. 45-60.

[DKLR] M. R. Douglas, R. L. Karp, S. LukiC and R. Reinbacher, Numerical solution to the Hermitian Yang-Mills equation on the Fermat quintic, J. High Energy Phys., 12 (2007), 083, $24 \mathrm{pp}$.

[KN] G. KempF And L. Ness, The length of vectors in representation spaces, in "Algebraic geometry (Proc. Summer Meeting, Univ. Copenhagen, Copenhagen, 1978)", pp. 233243, Lecture Notes in Math., 732, Springer, Berlin.

[NS] M. S. NARAsimhan AND C. S. Seshadri, Stable and unitary vectorbundles on a compact Riemann surface, Ann. of Math. (2), 82 (1965), pp. 540-567.

[PS] D. H. Phong and J. Sturm, Stability, energy functionals, and Kähler-Einstein metrics, Comm. Anal. Geom., 11:3 (2003), pp. 565-597.

[S] Y. SANo, Numerical algorithm for finding balanced metrics, Osaka J. Math., 43:3 (2006), pp. 679-688.

[UY] K. Uhlenbeck And S.-T. YAU, On the existence of Hermitian-Yang-Mills connections in stable vector bundles, Comm. Pure Appl. Math., 39:S (1986), suppl., pp. S257-S293.

[W] X. WANG, Balance point and stability of vector bundles over a projective manifold, Math. Res. Lett., 9:2-3 (2002), pp. 393-411.

[Zh] S. Zhang, Heights and reductions of semi-stable varieties, Compositio Math., 104:1 (1996), pp. 77-105. 
R. SEYYEDALI 